\title{
AN ELEMENTARY PROOF OF INTEGRATION BY PARTS FOR THE PERRON INTEGRAL
}

\section{LOUIS GORDON AND SIM LASHER}

In this note we give a proof of the theorem on integration by parts using the standard definition (see [2] or [3]) of the Perron integral in terms of major and minor functions. ${ }^{1}$

Theorem. Let $f$ be a Perron integrable function and $G$ a function of bounded variation on the finite interval $[a, b]$. Let

$$
F(x)=F(a)+(P) \int_{a}^{x} f d t, \quad a \leqq x \leqq b .
$$

Then $f G$ is Perron integrable on $[a, b]$ and

$$
\text { (P) } \left.\int_{a}^{b} f G d x=F(x) G(x)\right]_{a}^{b}-\int_{a}^{b} F d G,
$$

the last integral being Riemann-Stieltjes.

We may assume that $G(a)=F(a)=0$. Since every function $G$ of bounded variation vanishing at $x=a$ can be written as a linear combination of nondecreasing continuous functions and nondecreasing jump functions vanishing at $x=a$, it is sufficient to prove the theorem for these two types of functions. This is done in Lemmas 3 and 4 below.

Lemma 1. The theorem holds when $G$ is a nondecreasing jump function with a finite number of jumps and $G(a)=0$.

Proof. Since $G$ is the sum of a finite number of nondecreasing jump functions each having a single jump and vanishing at $x=a$, and since the lemma holds for each summand, it also holds for $G$.

Lemma 2. Let $f$ be a Perron integrable function on the finite interval $[a, b]$. Then there is a constant $k$ such that for every bounded nondecreas-

Received by the editors August 17, 1966.

1 It seems curious that a direct proof of the formula based only on the standard definition of the Perron integral does not exist in the literature (see the comment in $[2$, p. 101]). A proof based upon a definition of the Perron integral involving right and left major and minor functions is given in [4]. Proofs based upon the constructive and the descriptive definition of the special Denjoy integral-an equivalent of the Perron integral-are given respectively in [1] and [5]. 
ing function $G$ on $[a, b]$ with $G(a)=0$, there is a major function and $a$ minor function of $f G$ both of which are bounded in absolute value by $k G(b)$.

Proof. Let $k$ be a constant greater than $\operatorname{Osc}(F)$, the oscillation on $[a, b]$ of $F$, the indefinite Perron integral of $f$. Let $\psi$ be a major function of $f$ with $\operatorname{Osc}(\psi) \leqq k$. For $a \leqq u \leqq b$, let

$$
\begin{aligned}
\psi_{u}(x) & =\min [\psi(y): x \leqq y \leqq u] & & \text { if } a \leqq x \leqq u \\
& =\psi(x) & & \text { if } u \leqq x \leqq b .
\end{aligned}
$$

We will show that

$$
M(x)=\int_{a}^{b}\left[\psi_{u}(x)-\psi_{u}(a)\right] d G(u)
$$

is a major function of $f G$ and that

$$
|M(x)| \leqq k G(b), \quad a \leqq x \leqq b .
$$

Since $\psi_{u}(x)$ is (uniformly) continuous jointly in $x$ and $u, M(x)$ is continuous. Also $M(a)=0$. Let $a \leqq s \leqq t \leqq b$. Then

$$
\begin{aligned}
M(t)-M(s) & =\left(\int_{a}^{s}+\int_{s}^{t}+\int_{t}^{b}\right)\left[\psi_{u}(t)-\psi_{u}(s)\right] d G(u) \\
& \geqq[\psi(t)-\psi(s)] G(t) .
\end{aligned}
$$

Hence, for $x(a<x<b)$ a point of continuity of $G$, the lower derivate of $M$ at $x$

$$
D M(x) \geqq G(x) D \psi(x) .
$$

Thus

$$
D M(x)>-\infty \text { nearly everywhere }
$$

(that is, everywhere in $[a, b]$ with the possible exception of a denumerable subset of $[a, b])$; and

$$
D M(x) \geqq f(x) G(x) \text { a.e. }
$$

Also, since $\operatorname{Osc}\left(\psi_{u}\right) \leqq \operatorname{Osc}(\psi)$, (3) holds.

In parallel fashion, replacing in (2), $\psi, \psi_{u}$, min, respectively by $\phi$, $\phi_{u}$, max, where $\phi$ is a minor function of $f$ with $\operatorname{Osc}(\phi) \leqq k$, we obtain a minor function $m$ of $f G$ satisfying

$$
|m(x)| \leqq k G(b), \quad a \leqq x \leqq b .
$$

LEмMA 3. The theorem holds when $G$ is a nondecreasing bounded jump function such that $G(a)=0$. 
Proof. We may write $G=G_{1}+G_{2}$ with $G_{i}(i=1,2)$ a nondecreasing bounded jump function such that $G_{i}(a)=0, G_{1}$ having a finite number of jumps, and $G_{2}(b)$ arbitrarily small. By virtue of Lemmas 1 and 2 , there is a major function $M$ and a minor function $m$ of $f G$ such that $M(b)-m(b)$ is arbitrarily small. Hence $f G$ is Perron integrable. It follows from Lemma 2 that

$$
\left|(P) \int_{a}^{b} f G d x\right| \leqq k G(b),
$$

$k$ depending only upon $f$. Using (by Lemma 1) equation (1) for $G_{1}$, and inequality (4) for $G_{2}$, we see that

$$
\text { (P) } \int_{a}^{b} f G d x-F(b) G(b)+\int_{a}^{b} F d G
$$

is arbitrarily small and hence equal to zero.

LEMMA 4. The theorem holds for $G$ continuous and nondecreasing, and $G(a)=F(a)=0$.

Proof. Let $\psi$ and $\phi$ respectively be a major and a minor function of $f$ and let

$$
M(x)=\psi(x) G(x)-\int_{a}^{x} \phi d G .
$$

Then $M=M(x)$ is ${ }^{2}$ a major function of $f G$.

To begin with, $M$ is continuous and $M(a)=0$. Let $a<x<b$. Let $t>0$. We get

$$
\begin{aligned}
M(x+t)-M(x)= & G(x+t)[\psi(x+t)-\psi(x)] \\
& +[\psi(x)-\phi(x)][G(x+t)-G(x)] \\
& -\int_{x}^{x+t}[\phi(u)-\phi(x)] d G(u),
\end{aligned}
$$

and

$$
\begin{aligned}
\frac{M(x+t)-M(x)}{t} \geqq G(x+t) \frac{\psi(x+t)-\psi(x)}{t} \\
-\int_{x}^{x+t} \frac{\phi(u)-\phi(x)}{u-x}\left(\frac{u-x}{t}\right) d G(u) .
\end{aligned}
$$

Thus, the lower right derivate of $M$ at $x$

\footnotetext{
2 This form of $M$ was suggested by A. Zygmund.
} 


$$
D^{+} M(x)>-\infty \text { nearly everywhere. }
$$

Similarly for $t<0$,

$$
\begin{aligned}
\frac{M(x+t)-M(x)}{t}= & G(x+t)\left[\frac{\psi(x+t)-\psi(x)}{t}\right] \\
& +\int_{x+t}^{x} \frac{\psi(u)-\psi(x)}{u-x}\left(\frac{u-x}{t}\right) d G(u) \\
& -\frac{1}{t} \int_{x+t}^{x}[\psi(u)-\phi(u)] d G(u) ;
\end{aligned}
$$

and so the lower left derivate of $M$ at $x$

$$
D-M(x)>-\infty \text { nearly everywhere. }
$$

It follows from (6) and (7) that $D M(x)>-\infty$ nearly everywhere. Finally (as in [4]), for $t \neq 0$

$$
\begin{aligned}
\frac{M(x+t)-M(x)}{t}= & G(x)\left[\frac{\psi(x+t)-\psi(x)}{t}\right] \\
& +\frac{1}{t} \int_{x}^{x+t}[\psi(x+t)-\psi(u)] d G(u) \\
& +\frac{1}{t} \int_{x}^{x+t}[\psi(u)-\phi(u)] d G(u) \\
= & A+B+C .
\end{aligned}
$$

Now $C \geqq 0$. If $x$ is a point where $G$ has a finite derivative, then

$$
B=\left[\psi(x+t)-\psi\left(x_{0}\right)\right] \frac{G(x+t)-G(x)}{t} \rightarrow 0
$$

as $t \rightarrow 0, x_{0}$ being some point between $x$ and $x+t$. Therefore

$$
D M(x) \geqq G(x) f(x) \text { a.e. }
$$

Thus, $M$ is a major function of $f G$. Similarly it can be shown that

$$
m(x)=\phi(x) G(x)-\int_{a}^{x} \psi d G
$$

is a minor function of $f G$. Now

$$
M(b)-m(b)=[\psi(b)-\phi(b)] G(b)+\int_{a}^{b}(\psi-\phi) d G
$$


is small when $\psi(b)-\phi(b)$ is small. Hence $f G$ is Perron in tegrable; and letting $\psi$ and $\phi$ uniformly approach $F$, (1) follows from (5).

\section{REFERENCES}

1. E. W. Hobson, The theory of functions of a real variable and the theory of Fourier series, Vol. 1, 3rd ed., Cambridge Univ. Press, Cambridge, 1927, 711-715.

2. R. L. Jeffery, Non-absolutely convergent integrals, Proc. Second Canadian Math. Congress, pp. 93-145, Vancouver, 1949, Univ. of Toronto Press, Toronto, 1951.

3. E. Kampke, Das Lebesgue-Stieltjes-integral, 2nd ed., Teubner, Leipzig, 1960.

4. E. J. McShane, On Perron integration, Bull. Amer. Math. Soc. 48 (1942), 718-726.

5. S. Saks, Theory of the integral, 2nd rev. ed., Dover, New York, 1964, p. 246.

University of Illinois at Chicago Circle 